\title{
Imaging features in incident radiographic patellofemoral osteoarthritis: the Beijing Shunyi osteoarthritis (BJS) study
}

\author{
Yudian Qiu', Chutong Lin ${ }^{1}$, Qiang Liu', Qunjie Zhong ${ }^{1,2}$, Ke Tao $^{1}$, Dan Xing ${ }^{1}, \mathrm{Hu} \mathrm{Li}^{1}$ and Jianhao Lin ${ }^{1 *}$
}

\begin{abstract}
Background: The present study aims to describe the imaging features in incident radiographic patellofemoral osteoarthritis (RPFOA) population in a Chinese suburban area.

Methods: The Beijing Shunyi osteoarthritis (BJS) study was a population-based, longitudinal and prospective study. Residents were recruited by randomized cluster sampling in 2014 and were followed 3 years later. Home interviews and clinical examinations were performed; weight-bearing posterior-anterior semi-flexed (45-degree) views of the tibiofemoral (TF) joints and skyline (45-degree) views of the patellofemoral (PF) joints were included. For each batch of study films ( $n=100), 20$ films from the year 2014 and 20 previously read PF radiographs were fed back to test inter-/intra-reader repeatability. The imaging features of incident RPFOA were analyzed. Narrative statistics, independent-sample t-tests, and nonparametric tests were performed.
\end{abstract}

Results: A total of 1295 participants (2590 knees) were recruited at baseline in 2014, and 967 (74.7\%) residents were followed in 2017. Of all the knees $(n=1537)$ without RPFOA at baseline, 139 knees $(13.3 \%)$ across 119 people developed incident RPFOA. Compared with the whole population, age $(p=0.031)$, body mass index $(B M I, p=0.042)$, and incidence of knee pain symptoms $(p<0.01$ ) were significantly different in the incident RPFOA population, while range of motion ( $\mathrm{ROM}, p=0.052)$ and gender (0/1, $p=0.203$ ) showed no significance. In the incident population, the changes of each imaging indicator grade were evaluated —lateral patellofemoral osteophyte (LPOST, increased by 1.02), medial patellofemoral osteophyte (MPOST, increased by 0.49), lateral joint space narrowing (LSN, increased by 0.30 ), medial joint space narrowing (MJSN, increased by 0.06); indicator grade progress decreases, respectively. The progress of LPOST was the fastest among the four indicators $(p<0.01)$.

Conclusions: In this population-based longitudinal study, among the incident RPFOA population, the imaging indicators show that marginal patellofemoral osteophyte is more pronounced than patellofemoral joint space narrowing. LPOST is the fastest-progressing indicator among all the radiographic features, which is also the most common imaging manifestation of RPFOA. In the incident RPFOA population, the proportion of elders, women, higher-BMI individuals, and people suffering knee pain is more than the normal population.

Keywords: Patellofemoral osteoarthritis, Osteophyte, Joint space narrowing, Radiograph

\footnotetext{
* Correspondence: linjianhao@pkuph.edu.cn

${ }^{1}$ Institution of Arthritis, Peking University People's Hospital, NO11 South

street of Xizhimen, Xicheng district, Beijing, China

Full list of author information is available at the end of the article
}

(c) The Author(s). 2019 Open Access This article is distributed under the terms of the Creative Commons Attribution 4.0 International License (http://creativecommons.org/licenses/by/4.0/), which permits unrestricted use, distribution, and reproduction in any medium, provided you give appropriate credit to the original author(s) and the source, provide a link to the Creative Commons license, and indicate if changes were made. The Creative Commons Public Domain Dedication waiver (http://creativecommons.org/publicdomain/zero/1.0/) applies to the data made available in this article, unless otherwise stated. 


\section{Background}

Knee osteoarthritis (OA) is the most common joint disorders. It is a leading cause of pain and disability among adults $[1,2]$, impacting many health outcomes and leading to a high burden among the Chinese population [3]. Although osteoarthritis prevalence and incidence estimates have varied somewhat across studies [1, 4], there is agreement that a substantial proportion of adults are affected. The National Health Interview Survey estimated that 14 million people in the US have symptomatic knee OA [5].

Among knee OA patients, patellofemoral osteoarthritis (PFOA) is relatively common, which is one of the main causes of knee pain. Most studies investigating symptoms and disability in knee OA have defined OA as a disease in the tibiofemoral compartment. However, the disease is not limited to this compartment and may occur in the patellofemoral (PF) compartment, separately or in conjunction with the tibiofemoral compartment. Many factors can lead to osteophyte formation, cartilage damage, subchondral sclerosis, and PF joint subluxation. Female patients are more common in the PFOA population [6]. Previous literature had reported imaging evidence of PFOA in 17.1-34\% of women and $18.5-19 \%$ of men [7].

Since there are many definitions and classifications of radiographic patellofemoral osteoarthritis (RPFOA), no internationally recognized quantitative diagnostic criteria have been made. We defined a subject as having RPFOA on skyline view when (1) the osteophyte score was $\geq 2$; (2) the joint space narrowing (JSN) score was $\geq 2$ and the osteophyte score was $\geq 1$; (3) the JSN score was 3 , which refers to Felson and Baker et al. [8, 9], according to the International Association of Osteoarthritis (OARSI) atlas $[10,11]$ classification (grades $0-3 ; 0=$ normal, $1=$ mild, $2=$ moderate, $3=$ severe), RPFOA can be diagnosed if any criteria is met. In this study, the imaging features of incident RPFOA patients were analyzed from the aspects of PF joint space narrowing (medial / lateral) and PF joint osteophyte (medial / lateral).

\section{Methods}

The Beijing Shunyi osteoarthritis (BJS) study was a population-based, longitudinal, and prospective study of knee osteoarthritis. The BJS study was approved by the Ethics Committee of Peking University People's Hospital. Every resident who participated in the survey had signed the informed consent document. This study was based on a random cluster sampling method in 2014. Fourteen villages in Shunyi District, Beijing, China were selected. The inclusion criteria for this study were: (1) residence in Shunyi District, Beijing, and (2) age over 50 years. Exclusion criteria: (1) patients with rheumatoid arthritis, (2) physically disability, (3) mental retardation,
(4) patients with advanced malignant tumors or bedridden patients, and (5) people recently living or working outside for more than 6 months. And the rejection criteria: (1) people who did not meet the inclusion criteria, (2) people who met the exclusion criteria, (3) people who failed to complete the main records, and (4) people who showed obvious incompatibility in the survey. Respondents who met any of the criteria mentioned above were withdrawn from the survey.

\section{Clinical data collection}

A total of 1295 residents aged over 50 were enrolled in the baseline and were followed up the same month in 2017 as the BJS study. The baseline and follow-up surveys were standardized questionnaires completed at home or in the survey center. Imaging features (weightbearing posterior-anterior semi-flexed [45-degree] view of radiographs at TF joints and skyline [45-degree] view of radiographs at PF joints) were performed in local community hospitals. Clinical examinations including height, weight, range of motion (ROM), chair stand test, and 50 - $\mathrm{ft}$ walk test were performed by the same two clinicians in both 2014 and 2017. In this study, gender, age, height, body mass index (BMI), and knee joint ROM were selected, combined with the results of PF axial film, to summarize the distribution and characteristics of the incident RPFOA population.

\section{Imaging data analysis}

The imaging data in 2014 were read by two experienced researchers (one musculoskeletal radiologist [QZ] and one osteologist [HL], both blinded to the data). Independently, inconsistent data were checked by another skilled researcher [XL]. The imaging data in 2017 were read by a researcher who was blinded to the data and completed intra-reader repeatability to kappa ( $>0.8)$. For each batch of BJS study films $(n=100), 20$ films from the 2014 were added to test inter-reader repeatability. In addition, 20 previously read PF radiographs from 2017 study were fed back to test intra-reader repeatability. The diagnostic disease severity grade atlas of PF radiographic features on the skyline view is shown in Fig. 1.

\section{Statistical analysis}

Statistical analysis was done using SPSS (IBM, 25.0, CA, USA). The basic characteristics and clinical symptoms of the population were described by narrative statistics; independent-sample t-tests were performed to test the significance between the incident RPFOA patients and the whole population; and Kruskal-Wallis $\mathrm{H}$ tests were performed to compare results between the progress of lateral patellofemoral osteophyte (LPOST), medial patellofemoral osteophyte (MPOST), lateral joint space narrowing (LJSN), and medial joint space narrowing 

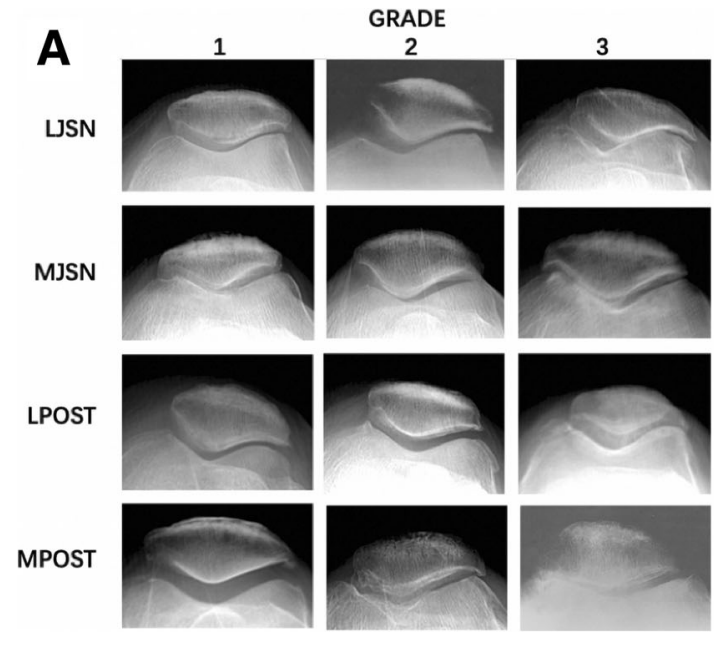

B
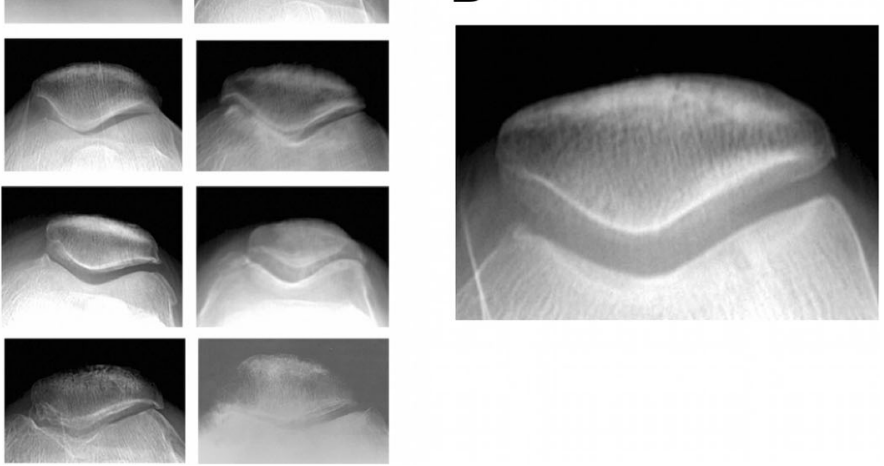

Fig. 1 The normal radiograph (b, grade 0) is contrasted to grade1, grade2, and grade 3 abnormal radiographic features (a) on the skyline view of the patellofemoral jointAbbreviations. LJSN: lateral joint space narrowing; MJSN: medial joint space narrowing; LPOST: lateral patellofemoral osteophyte; MPOST: medial patellofemoral osteophyte.

(M)SN). The results of analysis were presented as odds ratio (OR) with $95 \%$ confidence interval $(\mathrm{CI})$, and a $p$ value $<0.05$ was considered significant.

\section{Results}

\section{Descriptive characteristics}

A total of 1295 subjects were recruited at the baseline in 2014, and 967 (74.7\%) were followed in 2017. The BJS study population was predominantly female $(63.3 \%)$. The mean age was 60.3 years $(S D=6.60)$. The mean $B M I$ was 26.4 $(\mathrm{SD}=3.63)$. The mean knee $\mathrm{ROM}$ was $127.8 \mathrm{de}-$ grees $(\mathrm{SD}=9.24)$. A total of 117 subjects had knee pain symptoms (12.1\%) (Table 1$)$.

Among 1537 knee joint images without RPFOA at baseline, 139 cases (knee joint level) were diagnosed as incident RPFOA cases in 3 years. The incident RPFOA population was predominantly female (69.7\%). The mean age was 61.6 years $(\mathrm{SD}=6.44)$. The mean $\mathrm{BMI}$ was 27.2
$(\mathrm{SD}=3.99)$. The mean ROM of knee joint was 126.2 degrees $(\mathrm{SD}=8.1)$. A total of 21 incident RPFOA patients had knee pain symptoms (17.6\%) (Table 1). Compared with the whole population, age $(P=0.041)$, BMI $(P=$ 0.032 ), gender (male / female were valued $0 / 1, p<0.01$ ), and incidence of knee pain symptoms $(p<0.01)$ in incident RPFOA patients achieved significance, while ROM $(p=0.052)$ showed no significance.

\section{Imaging indicators measurement}

Unweighted $\mathrm{\kappa}$ coefficients for inter-reader and intrareader repeatability in 2017 were 0.82 and 0.85 , respectively. Among 139 incident RPFOA radiographs, the progress of the imaging indicator grade was analyzed. The progress of marginal PF osteophyte was more pronounced than joint space narrowing, while the mean grade progress of LPSOT is 1.02, showed significance when compared to that of MPOST

Table 1 Descriptive characteristics comparation between incident RPFOA patients and total population

\begin{tabular}{|c|c|c|c|}
\hline & Incident RPFOA Patients (119) & Total Population (967) & $P$ value \\
\hline Gender & & & $<0.01$ \\
\hline Male, n (\%) & $36(30.3)$ & $345(35.7)$ & \\
\hline Female, n (\%) & $83(69.7)$ & $622(63.3)$ & \\
\hline Age, years & & & 0.041 \\
\hline Mean (SD) & $61.6(6.44)$ & $60.3(6.60)$ & \\
\hline $\mathrm{BMI}$ & & & 0.032 \\
\hline Mean (SD) & $27.2(3.99)$ & $26.4(3.63)$ & \\
\hline $\mathrm{ROM}$ & & & 0.052 \\
\hline Mean (SD) & $126.2(8.10)$ & $127.8(9.24)$ & \\
\hline Knee pain, n (\%) & $21(17.6)$ & $117(12.1)$ & $<0.01$ \\
\hline
\end{tabular}


(0.49), LJSN (0.30), MJSN (0.06), and $p<0.01$. The composition of each imaging indicator grade progress of incident RPFOA is shown in Table 2. The comparison $p$-values regarding the progress between the four indicator grades are shown in Table 3.

\section{Discussion}

This is the first population-based, longitudinal, and prospective study demonstrating imaging features in the incident RPFOA population. Interestingly, we found that patella osteophyte progress faster than joint space narrowing. The progress of LPOST is significantly faster than the other three indicators, suggesting that PF joint lateral wear is the most common to be found in the incident RPFOA patients, and the formation of osteophyte is easier than that of joint space narrowing. PFOA is caused by the wear of patellar or trochlear groove cartilage, which is more common to be found in the lateral patellar region [12]. This indicates that the pressure on the lateral patella is greater than that on the central and medial patella. Baker et al. [9] found that quadriceps femoris weakness was more pronounced in patients with knee pain, and quadriceps femoris weakness was closely related to PFOA and tibiofemoral osteoarthritis (TFOA). Their study also showed that lateral compartment was more affected than medial compartment. When the knee buckles at 30 degrees, the abnormal pulley geometry reduces the lateral stability by $70 \%$, while the relaxation of the medial oblique femoris reduces the lateral stability by $30 \%$ [13]. There are complex interactions among these anatomical structures. They have different effects on lateral stability of PF joint in knee flexion. Their long-term effects may cause imaging changes of the PF joint.

However, the relationship between the imaging features of RPFOA and clinical symptoms and signs is not

Table 2 Imaging indicator grade progress of incident RPFOA in 3 years

Progress of Imaging Indicator Grades

\begin{tabular}{lllllllll}
\hline & 0 & 1 & & 2 & & 3 \\
LJSN, n (\%) & $108(77.7)$ & $0-1^{*}$ & $12(8.6)$ & $0-2^{*}$ & $8(5.8)$ & None \\
& & $1-2^{*}$ & $9(6.5)$ & $1-3^{*}$ & $2(1.4)$ & & \\
& & & & & \\
MJSN, n (\%) & $132(95.0)$ & $0-1^{*}$ & $6(4.3)$ & $0-2^{*}$ & $1(0.7)$ & None \\
LPOST, n (\%) & $35(25.2)$ & $0-1^{*}$ & $13(9.4)$ & $0-2^{*}$ & $36(25.9)$ & $0-3^{*}$ & $1(0.7)$ \\
& & $1-2^{*}$ & $54(38.8)$ & & & & \\
MPOST, n(\%) & $89(64.0)$ & $0-1^{*}$ & $19(13.7)$ & $0-2^{*}$ & $18(12.9)$ & None \\
& & $1-2^{*}$ & $13(9.4)$ & & & \\
\hline
\end{tabular}

*Indicates that the progress of each imaging indicator grade of the RPFOA radiographs increases from 0 to 1 (from 1 to 2, from 1 to 3 , or from 0 to 3 ) Abbreviations. LSSN lateral joint space narrowing; MJSN medial joint space narrowing; LPOST lateral patellofemoral osteophyte; MPOST medial patellofemoral osteophyte
Table 3 The p-values between the progress of imaging indicator grades of incident RPFOA

\begin{tabular}{llll}
\hline & MJSN (0.06) & LPOST (1.02) & MPOST (0.49) \\
\hline USN (0.30) & 0.01 & $<0.01$ & 0.114 \\
MJSN (0.06) & n.s. & $<0.01$ & $<0.01$ \\
LPOST (1.02) & n.s. & n.S. & $<0.01$
\end{tabular}

Abbreviations. n.s no significance; LJSN lateral joint space narrowing; MJSN medial joint space narrowing; LPOST lateral patellofemoral osteophyte; MPOST medial patellofemoral osteophyte

as typical as that of radiological tibial femoral arthritis (RTFOA). In our study, we found that the ROM in knee joint did not show significant difference between the incident RPFOA patients and the whole population. Although the incidence of knee joint pain was significantly different between the two groups, the rate of knee pain in incident RPFOA patients (17.6\%) was not as high as expected. A cohort study of 409 people by Camille Parsons et al. [14] demonstrated that clinical symptoms including knee bounce, patellar floating sign, knee tenderness and knee flexion pain were closely related to the increased risk of RTFOA, but only knee tenderness was associated with RPFOA. This is basically consistent with our findings, which may reflect that the contribution of imaging changes in isolated RPFOA are not as significant as the RTFOA in terms of the overall knee joint symptoms and physical signs. The symptoms of knee pain may be closely related to its overuse and injury history. Hip-muscle dysfunction, weakness of core muscle groups, excessive foot rotation, and patellar dislocation can also cause or aggravate knee pain symptoms [15]. Isolated RPFOA cannot be equated with knee joint symptoms.

Based on the results of our study, among all the incident RPFOA population, the proportion of women is higher than men, and the BMI of the incident RPFOA population is higher than that of the normal population. Besier et al. [16] found that quadriceps femoris muscle strength, especially medial muscle strength, plays an important role in maintaining patellar stability. In addition, muscle strength plays an important role in controlling the relative contact area between patella and femur under weight-bearing conditions. This may be the reason for the higher proportion of women. The study of Clark et al. [17] showed that the relative contact area between patella and femur increased by $38 \%$ when the muscle strength of medial femoral muscle increased from $0 \mathrm{~N}$ to $100 \mathrm{~N}$ at 30-degree knee flexion. This demonstrates that greater medial femoral muscle strength can lead to the increasement of the PF contact area; thereby the stability of the PF joint can be increased and the wear of the PF joint can be reduced. The pressure of the knee compartment is higher in people with higher BMI, which makes it more likely to form imaging progresses of the PF joint. 
The diagnosis of RPFOA is usually interpreted by skyline view (45-degree) of the patellofemoral (PF) joints [18]. The skyline view is more accurate than lateral radiographs in reading joint space narrowing and patella osteophyte $[19,20]$. With this information in mind, all the RPFOA diagnoses in the BJS study were based on skyline view for higher accuracy. The view chosen to define RPFOA may be critical [21]. A limitation of our study was that there was no gold standard for RPFOA diagnosis in international guidelines. Also, studies often have incomplete views of the knee joint. When the views are extended to encompass all aspects of the knee joint, the prevalence of radiographic changes of osteoarthritis may be higher. Because of the needs of our study, we redefined RPFOA based on definitions in previous articles $[8,9]$ by OARSI and its atlas. We consider that this definition to be feasible and to have a quantitative standard in clinical practice.

There are still other limitations of our study. Firstly, the population measurement in this study was localized in the Beijing area, which could have possibly influenced the precision of the results. Secondly, due to the relatively small population in Beijing engaged in heavy manual labor, the number of incident RPFOA cases may have been underestimated. Third, the follow-up duration of our study was 3 years, which was relatively shorter than in other studies. Given that the development of RPFOA is unlikely to occur in a short time, a longer period of observation is required to validate our findings. Fourth, radiographs cannot detect minor osteoarthritis changes when compared with magnetic resonance imaging (MRI) studies. We chose radiographs because they were easier to operate and relatively inexpensive. In addition, this study did not further classify isolated RPFOA and mixed (RPFOA + RTFOA) knee osteoarthritis, which are difficult to gauge as accurate reflections of the imaging features of RPFOA in the general population.

\section{Conclusions}

RPFOA is often accompanied by knee pain and other symptoms. Although it is not as closely associated with knee joint symptoms as RTFOA, it still affects people's quality of life to a great extent. Women, older people, and people with high BMI and knee pain compose the incident RPFOA population in greater proportion than in the overall population. In this study, the imaging features of incident RPFOA are briefly described: PF osteophyte is more pronounced than PF joint space narrowing, and LPOST is the most common imaging manifestation of RPFOA, with the fastest progress in severity grade. We hope that this study will enable people to have a clearer understanding of PFOA and to make efforts toward greater public health in China.

\section{Abbreviations}

BMl: Body mass index; LJSN: Lateral joint space narrowing; LPOST: Lateral patellofemoral osteophyte; MJSN: Medial joint space narrowing; MPOST: Medial patellofemoral osteophyte; MRI: Magnetic resonance imaging; OA: Osteoarthritis; OARSI: Osteoarthritis Research Society International; PF: Patellofemoral; PFOA: Patellofemoral osteoarthritis; ROM: Range of motion; RPFOA: Radiographic patellofemoral osteoarthritis;

RTFOA: Radiographic tibiofemoral osteoarthritis; TF: Tibiofemoral

\section{Acknowledgements}

We thank Dr. Yuqing Zhang for his help with statistics analysis methods. We thank Dr. Xiaojuan Li for her help in the reading of patellofemoral radiographs.

\section{Authors' contributions}

$J \mathrm{~L}, \mathrm{YQ}$, and $\mathrm{HL}$ designed the study and analyzed the data. $\mathrm{YQ}$ and $\mathrm{CL}$ wrote the manuscript. YQ, CL, QL, DX, QZ, KT, HL, and JL participated in the data collection, analysis, and interpretation. All authors read and approved the final manuscript.

\section{Funding}

This investigation was supported in part by the National Natural Science Foundation of China (NSFC), Grant/Award Number 81501919/81672183. This funding was not involved in the data collection, data analysis, or the preparation or editing of the manuscript.

\section{Availability of data and materials}

Data will be available upon request by the first author YQ.

\section{Ethics approval and consent to participate}

This study was approved by the ethics committee of the Peking University People's Hospital. All patients provided written informed consent prior to their inclusion in this study.

\section{Consent for publication \\ Not applicable.}

\section{Competing interests}

The authors declare that they have no competing interests.

\section{Author details}

${ }^{1}$ Institution of Arthritis, Peking University People's Hospital, NO11 South street of Xizhimen, Xicheng district, Beijing, China. ${ }^{2}$ Department of Radiology and Biomedical Imaging, University of California, San Francisco, San Francisco, CA, USA.

Received: 11 May 2019 Accepted: 19 July 2019

Published online: 07 August 2019

References

1. Neogi T, Zhang Y. Epidemiology of osteoarthritis. Rheum Dis Clin N Am. 2013;39:1e19.

2. McDonough $C M$, Jette $A M$. The contribution of osteoarthritis to functional limitations and disability. Clin Geriatr Med Aug. 2010;26:387-99.

3. Qiang $L$, Shengfeng W, Yuqing Z. The burden for knee osteoarthritis among Chinese elderly: estimates from a nationally representative study. Osteoarthr Cartil. 2018;26:1636-42.

4. Johnson VL, Hunter DJ. The epidemiology of osteoarthritis. Best Pract Res Clin Rheumatol. 2014;28:5-15.

5. Deshpande BR, Katz JN, Solomon DH, et al. Number of persons with symptomatic knee osteoarthritis in the US: impact of race and ethnicity, age, sex, and obesity. Arthritis Care Res. 2016;68:1743-50.

6. Duncan R, Peat G, Thomas $E$, et al. Incidence, progression and sequence of development of radiographic knee osteoar- thritis in a symptomatic population. Ann Rheum Dis. 2011;70(11):1944-8.

7. Davies AP, Vince AS, Shepstone $L$, et al. The radiologic prevalence of patellofemoral osteoarthritis. Clin Orthop Relat Res. 2002;402:206-12.

8. Felson DT, Nevitt MC, Yang M, et al. A new approach yields high rates of radiographic progression in knee osteoarthritis. J Rheumatol. 2008; 35(10):2047. 
9. Baker KR, Xu L, Zhang Y, et al. Quadriceps weakness and its relationship to tibiofemoral and patellofemoral knee osteoarthritis in Chinese: the Beijing osteoarthritis study. Arthritis Rheum. 2010;50(6):1815-21.

10. Altman RD, Hochberg M, Murphy WA Jr, et al. Atlas of individual radiographic features in osteoarthritis. Osteoarthritis Cartilage. 1995; 3((Suppl A):3-70.

11. Hart DJ, Spector TD. The classification and assessment of osteoarthritis. Baillieres Clin Rheumatol. 1995;9(2):407-32.

12. Saleh KJ, Elizabeth $A$, et al. Symposium: operative treatment of patellofemoral arthritis. J Bone Joint Surg (Am Vol). 2005;87(3):659-71.

13. Senavongse $W$, Amis $A$. The effects of articular, retinacular, or muscular deficiencies on patellofemoral joint stability [J]. J Bone Joint Surg Br Vol. 2005;87(4):577.

14. Parsons C, Fuggle NR, Edwards MH, et al. Concordance between clinical and radiographic evaluations of knee osteoarthritis [J]. Aging Clin Exp Res. 2018; 30(1):17-25.

15. Farzin $H$, Maryam A, Maryam M, et al. Patellofemoral pain in athletes: clinical perspectives. Open Access J of Sports Medicine. 2017;8:189-203.

16. Besier TF, Draper CE, Gold GE, et al. Patellofemoral joint contact area increases with knee flexion and weight-bearing.[J]. J Orthop Res. 2010; 23(2):345-50,

17. Clark AL, Herzog W, Leonard TR. Contact area and pressure distribution in the feline patellofemoral joint under physiologically meaningful loading conditions. J Biomech. 2002;35:53-60.

18. Heng HYC, Razak HRBA, Mitra AK. Radiographic grading of the patellofemoral joint is more accurate in skyline compared to lateral views [J]. Ann Transl Med. 2015;3(18):263.

19. McDonnell SM, Bottomley NJ, Hollinghurst D, et al. Skyline patellofemoral radiographs can only exclude late stage degenerative changes. Knee. 2011;18:21-3.

20. Collins DH, Mcelligott TF. Sulphate (35SO4) uptake by chondrocytes in relation to histological changes in Osteo-arthritic human articular cartilage [J]. Ann Rheum Dis. 1960;19(4):318-30

21. Duncan RC. Prevalence of radiographic osteoarthritis--it all depends on your point of view [J]. Rheumatology. 2006;45(6):757-60.

\section{Publisher's Note}

Springer Nature remains neutral with regard to jurisdictional claims in published maps and institutional affiliations.

Ready to submit your research? Choose BMC and benefit from:

- fast, convenient online submission

- thorough peer review by experienced researchers in your field

- rapid publication on acceptance

- support for research data, including large and complex data types

- gold Open Access which fosters wider collaboration and increased citations

- maximum visibility for your research: over $100 \mathrm{M}$ website views per year

At $\mathrm{BMC}$, research is always in progress.

Learn more biomedcentral.com/submissions 